\title{
Note on the Zolotarev optimal rational approximation for the overlap Dirac operator
}

\author{
Ting-Wai Chiu, * Tung-Han Hsieh, Chao-Hsi Huang, and Tsung-Ren Huang \\ Department of Physics, National Taiwan University, Taipei, Taiwan 106, Taiwan
}

(Received 12 June 2002; published 27 December 2002)

\begin{abstract}
We discuss the salient features of the Zolotarev optimal rational approximation for the inverse square root function, in particular, for its applications in lattice QCD with the overlap Dirac quark. The theoretical error bound for the matrix-vector multiplication $H_{w}\left(H_{w}^{2}\right)^{-1 / 2} Y$ is derived. We check that the error bound is always satisfied amply, for any QCD gauge configurations we have tested. An empirical formula for the error bound is determined, together with its numerical values (by evaluating elliptic functions) listed in Table II as well as plotted in Fig. 3. Our results suggest that, with the Zolotarev approximation to $\left(H_{w}^{2}\right)^{-1 / 2}$, one can essentially preserve the exact chiral symmetry of the overlap Dirac operator to very high precision, for any gauge configuration on a finite lattice.
\end{abstract}

DOI: 10.1103/PhysRevD.66.114502

PACS number(s): 11.15.Ha, 11.30.Rd, 12.38.Gc

\section{INTRODUCTION}

In lattice QCD with overlap Dirac quarks [1,2], one encounters the challenging problem of taking the inverse square root of a positive definite Hermitian matrix, which stems from the overlap Dirac operator for a massless fermion,

$$
D=m_{0} a^{-1}\left(1+\gamma_{5} \frac{H_{w}}{\sqrt{H_{w}^{2}}}\right),
$$

where $H_{w}$ denotes the Hermitian Wilson-Dirac operator with a negative parameter $-m_{0}$,

$$
H_{w}=\gamma_{5} D_{w}=\gamma_{5}\left(-m_{0}+\gamma_{\mu} t_{\mu}+W\right)
$$

$\gamma_{\mu} t_{\mu}$ the naive fermion operator, and $W$ the Wilson term. It is well known that Eq. (1) is the simplest and most elegant realization of chiral symmetry on a finite lattice [3],

$$
D \gamma_{5}+\gamma_{5} D=a m_{0}^{-1} D \gamma_{5} D
$$

and it also satisfies all basic requirements (i.e., doublers-free, $\gamma_{5}$ hermiticity, exponentially local, and correct continuum behavior) for a decent lattice Dirac operator. However, in practice, how to compute physical quantities in lattice QCD with an overlap Dirac operator (1) is still a challenging problem, due to the inverse square root of $H_{w}^{2}$ in Eq. (1), which cannot be evaluated analytically in a closed form, nor numerically via diagonalization since the memory requirement exceeds the physical memory of the present generation of computers. Thus, at this stage, it is necessary to replace $\left(H_{w}^{2}\right)^{-1 / 2}$ with a good approximation, in any lattice QCD calculations with overlap Dirac quarks. The relevant question is what is the optimal rational approximation $r_{n}(x)$ (of degree $n$ ) for the inverse square root function $x^{-1 / 2}, x \in[1, b]$, since the convergence of a rational approximation can attain

\footnotetext{
*Email address: twchiu@phys.ntu.edu.tw
}

$$
\max _{1 \leqslant x \leqslant b}\left|1-\sqrt{x} r_{n}(x)\right|=c_{1} e^{-c_{2} n}, \quad c_{1}, c_{2}>0
$$

which is much faster than that of any polynomial approximation. It turns out that the optimal solution was obtained by Zolotarev [4] more than 100 years ago. A detailed discussion of Zolotarev's result can be found in Akhiezer's two books $[5,6]$. Unfortunately, Zolotarev's optimal rational approximation was overlooked by the numerical algebra community until recent years. ${ }^{1}$

A comparative study of Zolotarev's approxiamtion versus other schemes for computing the product of the matrix sign function $H_{w}\left(H_{w}^{2}\right)^{-1 / 2}$ with a vector $Y$ has been reported in Ref. [8].

Recently, we used the Zolotarev optimal rational approximation to compute the overlap Dirac quark propagator in the quenched approximation [9]. The parameters of the pseudoscalar meson mass formula to one-loop order in chiral perturbation theory are determined, and from these the light quark masses $m_{u, d}$ and $m_{s}$ can be extracted [10] with the experimental inputs of pion and kaon masses, and the pion decay constant.

Nevertheless, in Refs. [8,9], the underlying principles and salient features ${ }^{2}$ of the Zolotarev optimal rational approximation were not addressed. Also, in Refs. [7-9], only one option [Eq. (70)] of the two possible choices [Eqs. (29) and (70)] of Zolotarev's rational polynomials was exploited, but it turns out that the other option [Eq. (29)] is more effective for the inverse square root of a matrix, as discussed in Sec. V.

In this paper, we discuss the salient features of the Zolotarev optimal rational approximation, in particular, for its application in lattice QCD with the overlap Dirac operator. We start with the basic principles of rational approximation, and show that Zolotarev's rational polynomial is indeed the

\footnotetext{
${ }^{1}$ See, for example, Ref. [7].

${ }^{2}$ In particular, the theoretical error bound for the product $H_{w}\left(H_{w}^{2}\right)^{-1 / 2} Y$ can be derived in terms of the deviation (35), as shown in Sec. V. Moreover, we clarify that the coefficients $d_{0}$ [Eq. (49)] and $D_{0}$ [Eq. (74)] can be obtained explicitly, without using the condition $\left.\max \left[1-\sqrt{x} r_{n}(x)\right]\right|_{1 \leqslant x \leqslant b}=-\left.\min \left[1-\sqrt{x} r_{n}(x)\right]\right|_{1 \leqslant x \leqslant b}$.
} 
optimal rational approximation for the inverse square root function. Then we examine how well the Zolotarev optimal rational approximation [Eq. (48)] for the overlap Dirac operator can preserve the exact chiral symmetry (3) on a finite lattice. For the overlap Dirac operator, Eq. (3) is equivalent to

$$
\left(\frac{H_{w}}{\sqrt{H_{w}^{2}}}\right)^{2}=1
$$

Thus the question is how much the exact relation (5) is violated if one replaces $\left(H_{w}^{2}\right)^{-1 / 2}$ with Zolotarev's optimal rational polynomial $r_{Z}^{(n)}\left(H_{w}^{2}\right)$. The chiral symmetry breaking can be measured in terms of the deviation

$$
\Delta_{Z}=\max _{\forall Y \neq 0}\left|\frac{Y^{\dagger}\left\{H_{w} r_{Z}^{(n)}\left(H_{w}^{2}\right)\right\}^{2} Y}{Y^{\dagger} Y}-1\right| .
$$

It turns out that the deviation (6) has a theoretical upper bound (62), which is a function of $n$ (the degree of the rational polynomial), and $b=\lambda_{\max }^{2} / \lambda_{\min }^{2}$, where $\lambda_{\max }^{2}$ and $\lambda_{\min }^{2}$ denote the maximum and the minimum of the eigenvalues of $H_{w}^{2}$. Thus, for any given gauge configuration, one can use the theoretical upper bound to determine what values of $n$ and $b$ (i.e., how many low-lying eigenmodes of $H_{w}^{2}$ should be projected out) are required to attain one's desired accuracy in preserving the chiral symmetry of the overlap Dirac operator. In practice, one has no difficulties in achieving $\Delta_{Z}<10^{-12}$ for any gauge configuration on a finite lattice.

The outline of this paper is as follows. In Secs. II-IV, we review the basic principles of rational approximation, and show that Zolotarev's rational polynomial is indeed the optimal rational approximation for the inverse square root function. In Sec. V, we discuss the Zolotarev approximation for $\left(H_{w}^{2}\right)^{-1 / 2}$ in the overlap Dirac operator, and derive the theoretical error bound for the matrix-vector multiplication $H_{w}\left(H_{w}^{2}\right)^{-1 / 2} Y(Y$ is any nonzero column vector), which is the most essential operation in computing the propagator of the overlap Dirac quark using the nested conjugate gradient. We check that the theoretical error bound is always satisfied amply, for any QCD gauge configurations we have tested. Then the numerical values of the theoretical error bound are computed, and listed in Table II, as well as plotted in Fig. 3. An empirical formula for the error bound is determined from the numerical data. In Sec. VI, we conclude with some remarks.

\section{DE LA VALLÉE-POUSSIN'S THEOREM}

First we consider the following problem. Given any two positive and continuous functions $f(x)$ and $g(x)$ for $x$ $\in[1, b]$, the problem is to find the irreducible rational polynomial of degree $n$ with positive real coefficients,

$$
r(x)=\frac{p(x)}{q(x)}=\frac{p_{n} x^{n}+p_{n-1} x^{n-1}+\cdots+p_{0}}{q_{n} x^{n}+q_{n-1} x^{n-1}+\cdots+q_{0}},
$$

such that the deviation of $g(x) r(x)$ from $f(x)$ is the minimum. Here the deviation is defined as the maximum of $|f(x)-g(x) r(x)|$ for the entire interval [1,b], i.e.,

$$
d(r)=\max _{x \in[1, b]}|f(x)-g(x) r(x)| .
$$

Now if there exists $r(x)$ such that the difference

$$
\delta(x)=f(x)-g(x) r(x)
$$

changes sign alternately $2 n+2$ times within the interval $[1, b]$, and attains its maxima and minima, say,

$$
\delta(x)=\delta_{1},-\delta_{2}, \ldots,+\delta_{2 n+1},-\delta_{2 n+2}\left(\delta_{i}>0\right),
$$

at consecutive points

$$
x_{1}<x_{2}<\cdots<x_{2 n+2},
$$

then it can be shown that

$$
d(r) \geqslant \min \left\{\delta_{1}, \delta_{2}, \ldots, \delta_{2 n+2}\right\} \equiv d_{\min }
$$

for all irreducible rational polynomial $r(x)$ as defined by Eq. (7). This can be asserted as follows.

The strategy is to assume the contrary is true, and then show that it leads to a contradiction. Suppose there exists an irreducible rational polynomial $R(x)$ of degree $n$, such that $d(R)<d_{\min }$. Then

$$
\begin{aligned}
D(x) & \equiv g(x) R(x)-g(x) r(x) \\
& =f(x)-g(x) r(x)-[f(x)-g(x) R(x)] \\
& =\delta(x)-\Delta(x)
\end{aligned}
$$

is a continuous function, and it also changes sign alternately at least $2 n+2$ times in the interval $[1, b]$, since $|\Delta(x)|$ $\leqslant d(R)<d_{\min }$. Thus $D(x)$ has at least $2 n+1$ zeros in the interval $(1, b)$. On the other hand,

$$
D(x)=g(x)[R(x)-r(x)] \equiv g(x) \frac{u(x)}{v(x)}
$$

where $u(x)$ and $v(x)$ are polynomials of degree $2 n$. Therefore $D(x)$ cannot have more than $2 n$ zeros, i.e., a contradiction. This completes the proof of de la Vallée-Poussin's theorem.

In general, de la Vallée-Poussin's theorem [5] asserts that if there exists an irreducible rational polynomial of the form

$$
r^{(n, m)}(x)=\frac{p_{n} x^{n}+p_{n-1} x^{n-1}+\cdots+p_{0}}{q_{m} x^{m}+q_{m-1} x^{m-1}+\cdots+q_{0}}\left(m \geqslant n, p_{i}, q_{i}>0\right)
$$

such that $\delta(x)=f(x)-g(x) r^{(n, m)}(x)$ has $n+m+2$ alternate changes of sign in the interval $[1, b]$, and attains its maxima and minima, say,

$$
\delta(x)=\delta_{1},-\delta_{2}, \ldots,(-1)^{n+m+1} \delta_{n+m+2}\left(\delta_{i}>0\right),
$$


at consecutive points

$$
x_{1}<x_{2}<\cdots<x_{n+m+2},
$$

then

$$
d(r) \geqslant \min \left\{\delta_{1}, \delta_{2}, \ldots, \delta_{n+m+2}\right\} \equiv d_{\text {min }}
$$

for all irreducible rational polynomials $r^{(n, m)}(x)$.

In the next section, we show that there exists an optimal $r_{Z}(x)$ such that $d\left(r_{Z}\right)$ is minimum, i.e., Zolotarev's solution [4].

\section{ZOLOTAREV'S OPTIMAL RATIONAL APPROXIMATION}

First, we recall some well-known properties of Jacobian elliptic functions [6]. The Jacobian elliptic function $\operatorname{sn}(u ; \kappa)=\eta$ is defined by the elliptic integral

$$
u(\eta)=\int_{0}^{\eta} \frac{d t}{\sqrt{\left(1-t^{2}\right)\left(1-\kappa^{2} t^{2}\right)}}
$$

It is a meromorphic function of $u$ and $\kappa$, which is defined over $C^{2}$. For $\eta=1$, Eq. (16) becomes

$$
u(1) \equiv K=\int_{0}^{1} \frac{d t}{\sqrt{\left(1-t^{2}\right)\left(1-\kappa^{2} t^{2}\right)}}
$$

the complete elliptic integral of the first kind with modulus $\kappa$.

The important feature of $\operatorname{sn}(u ; \kappa)$ is that it is periodic in both real and imaginary parts of $u$,

$$
\begin{array}{r}
\operatorname{sn}(u+4 K ; \kappa)=\operatorname{sn}(u ; \kappa), \\
\operatorname{sn}\left(u+2 i K^{\prime} ; \kappa\right)=\operatorname{sn}(u ; \kappa),
\end{array}
$$

where $K^{\prime}$ is a complete elliptic integral of the first kind with modulus $\kappa^{\prime}=\sqrt{1-\kappa^{2}}$.

Now if we define

$$
x(u ; \kappa) \equiv \operatorname{sn}^{2}(u ; \kappa),
$$

then $x$ has periods $2 K$ and $2 i K^{\prime}$, since $\operatorname{sn}(u+2 K ; \kappa)=$ $-\operatorname{sn}(u ; \kappa)$.

The crucial formula for Zolotarev's optimal rational approximation is the second principal $n$th degree transformation of the Jacobian elliptic function [6]. In terms of $x$ [Eq. (20)], it reads

$$
\sqrt{x\left(\frac{u}{M} ; \lambda\right)}=\sqrt{x(u ; \kappa)} \frac{1}{M} \prod_{l=1}^{n} \frac{1+x(u ; \kappa) / c_{2 l}}{1+x(u ; \kappa) / c_{2 l-1}}
$$

where

$$
\lambda=\prod_{l=1}^{2 n+1} \frac{\Theta^{2}\left(2 l K^{\prime} /(2 n+1) ; \kappa^{\prime}\right)}{\Theta^{2}\left((2 l-1) K^{\prime} /(2 n+1) ; \kappa^{\prime}\right)},
$$

$$
M=\prod_{l=1}^{n} \frac{\operatorname{sn}^{2}\left((2 l-1) K^{\prime} /(2 n+1) ; \kappa^{\prime}\right)}{\operatorname{sn}^{2}\left(2 l K^{\prime} /(2 n+1) ; \kappa^{\prime}\right)},
$$

$$
c_{l}=\frac{\operatorname{sn}^{2}\left(l K^{\prime} /(2 n+1) ; \kappa^{\prime}\right)}{1-\operatorname{sn}^{2}\left(l K^{\prime} /(2 n+1) ; \kappa^{\prime}\right)},
$$

and $\Theta$ denotes the elliptic theta function. Note that $x(u / M ; \lambda)$ has periods $2 L=2 K / M$ and $2 i L^{\prime}=2 i K^{\prime} /[(2 n$ $+1) M]$

$$
\begin{aligned}
x\left(\frac{u+2 K}{M} ; \lambda\right) & =x\left(\frac{u}{M} ; \lambda\right), \\
x\left(\frac{u}{M}+\frac{2 i K^{\prime}}{(2 n+1) M} ; \lambda\right) & =x\left(\frac{u}{M} ; \lambda\right) .
\end{aligned}
$$

Now restricting $u=K+i v$, we have

$$
x(u ; \kappa)=\operatorname{sn}^{2}(u ; \kappa)=\frac{1-\operatorname{sn}^{2}(i v ; \kappa)}{1-\kappa^{2} \operatorname{sn}^{2}(i v ; \kappa)}=\frac{1}{1-\kappa^{\prime 2} \operatorname{sn}^{2}\left(v ; \kappa^{\prime}\right)},
$$

where the identity

$$
\operatorname{sn}^{2}(i v ; \kappa)=-\frac{\operatorname{sn}^{2}\left(v ; \kappa^{\prime}\right)}{1-\operatorname{sn}^{2}\left(v ; \kappa^{\prime}\right)}
$$

has been used. Equation (27) implies that $x$ increases from 1 to $1 / \kappa^{2}$ as $v$ increases from 0 to $K^{\prime}$, since $\operatorname{sn}\left(0 ; \kappa^{\prime}\right)=0$ and $\operatorname{sn}\left(K^{\prime} ; \kappa^{\prime}\right)=1$, from the definition (16).

Now consider

$$
r_{Z}(x)=\frac{2 \lambda}{1+\lambda} \frac{1}{M} \prod_{l=1}^{n} \frac{1+x / c_{2 l}}{1+x / c_{2 l-1}}
$$

as a rational approximation to $1 / \sqrt{x}$. Then the deviation can be obtained from

$$
\delta_{Z}(x)=1-\sqrt{x} r_{Z}(x)=1-\frac{2 \lambda}{1+\lambda} \operatorname{sn}\left(\frac{K+i v}{M} ; \lambda\right)
$$

where Eq. (21) has been used. Using the identity (27) with substitutions $\kappa \leftarrow \lambda$ and $u \leftarrow(K+i v) / M$, Eq. (30) becomes

$$
\delta_{Z}(x)=1-\frac{2 \lambda}{1+\lambda} \frac{1}{\sqrt{1-\lambda^{\prime 2} \operatorname{sn}^{2}\left(v / M ; \lambda^{\prime}\right)}},
$$

where $\lambda^{\prime}=\sqrt{1-\lambda^{2}}$. As $v$ changes from 0 to $K^{\prime}, v / M$ changes from 0 to $K^{\prime} / M=(2 n+1) L^{\prime}$. This implies that $\operatorname{sn}^{2}\left(v / M ; \lambda^{\prime}\right)$ attains its minima and maxima alternately as $0,1, \ldots, 0,1$ at $v / M=0, L^{\prime}, \ldots, 2 n L^{\prime},(2 n+1) L^{\prime}$. Correspondingly, $\delta_{Z}(x)$ reaches its maxima and minima alternately as

$$
\frac{1-\lambda}{1+\lambda},-\frac{1-\lambda}{1+\lambda}, \cdots, \frac{1-\lambda}{1+\lambda},-\frac{1-\lambda}{1+\lambda}
$$




$$
1-r_{z}(x) x^{1 / 2}
$$

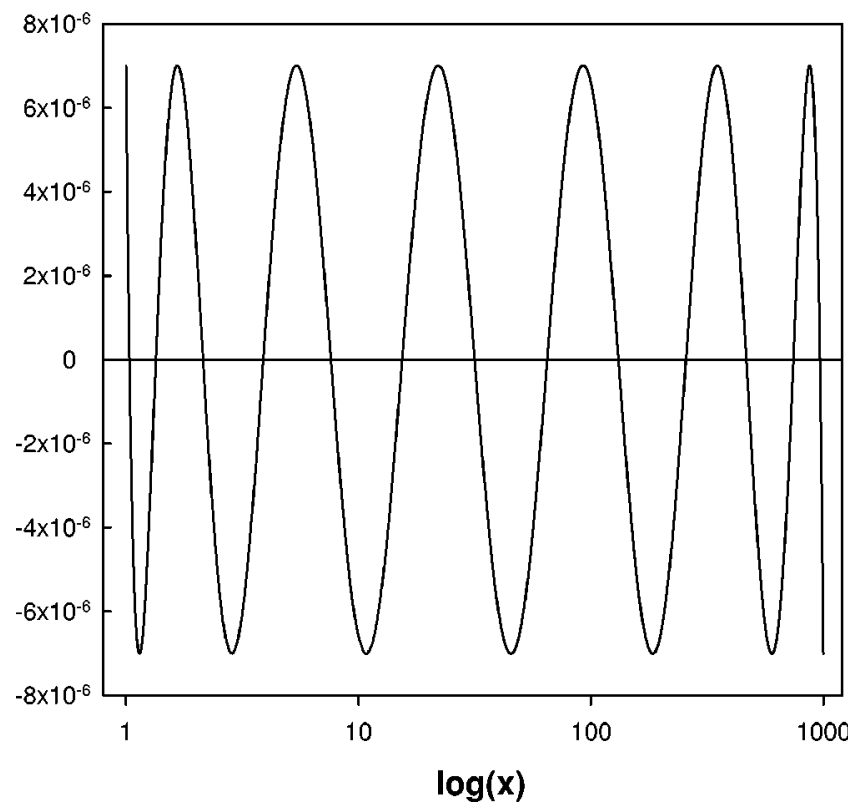

FIG. 1. The difference $1-\sqrt{x} r_{Z}(x)$ of the Zolotarev optimal rational approximation (29) with $n=6$ and $b=\kappa^{-2}=1000$. Note that there are exactly $2 n+2=14$ alternate changes of sign in $[1,1000]$, and the maxima and minima have exactly the same magnitude.

at $v / M=0, L^{\prime}, \ldots, 2 n L^{\prime},(2 n+1) L^{\prime}$, which correspond to $2 n+2$ successive $x \in\left[1,1 / \kappa^{2}\right]$,

$$
\begin{aligned}
& 1, \frac{1}{1-\kappa^{\prime 2} \operatorname{sn}^{2}\left(K^{\prime} /(2 n+1) ; \kappa^{\prime}\right)}, \\
& \cdots, \frac{1}{1-\kappa^{\prime 2} \operatorname{sn}^{2}\left(2 n K^{\prime} /(2 n+1) ; \kappa^{\prime}\right)}, \frac{1}{\kappa^{2}} .
\end{aligned}
$$

That is,

$$
\begin{aligned}
x_{i}= & \frac{1}{1-\kappa^{\prime 2} \operatorname{sn}^{2}\left((i-1) K^{\prime} /(2 n+1) ; \kappa^{\prime}\right)}, \\
& i=1, \ldots, 2 n+2 .
\end{aligned}
$$

From Eq. (32), we conclude that

$$
d\left(r_{Z}\right)=\frac{1-\lambda}{1+\lambda}=d_{\min },
$$

and Zolotarev's solution (29) is the optimal rational approximation for the inverse square root function, according to de la Vallée-Poussin's theorem.

At this point, it is instructive to plot $\delta_{Z}(x)=1$ $-\sqrt{x} r_{Z}(x)$ explicitly, and to see how it attains its maxima and minima alternately. This is shown in Fig. 1 for $n=6$ and $b=\kappa^{-2}=1000$. Note that there are exactly $2 n+2=14$ alternate changes of sign in $[1,1000]$, with maxima and minima at

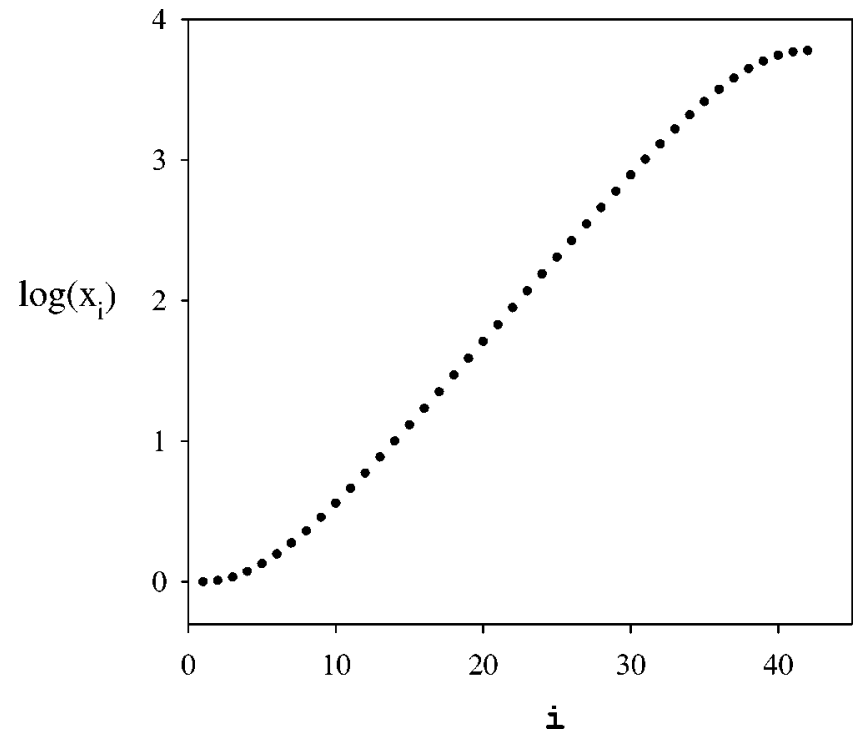

FIG. 2. The positions $x_{i}$ [Eq. (34)] of the maxima and minima of $1-\sqrt{x} r_{Z}(x)$ for $n=20$ and $b=\kappa^{-2}=6000$.

$$
x=1,1.145,1.664,2.858,5.415,10.80,22.05,45.34,
$$

$$
92.59,184.7,349.9,600.9,873.3,1000 \text {. }
$$

In Fig. 2, we plot the positions $x_{i}$ [Eq. (34)] of the maxima and minima of $\delta_{Z}(x)=1-\sqrt{x} r_{Z}(x)$ for $n=20$ and $b=\kappa^{-2}=6000$. Note that the distribution of $x_{i}$ as shown in Fig. 2 is quite generic for any values of $n$ and $b$. The maxima and minima near both ends $\left(x_{1}=1\right.$ and $\left.x_{2 n+2}=b\right)$ are densely packed even on the logarithmic scale, while those at the central region seem to be evenly distributed on the log scale (i.e., exponentially on the linear scale).

\section{CHEBYSHEV'S THEOREM}

Even though Zolotarev's rational approximation is optimal among all irreducible rational polynomials of degree $n$ with $\delta(x)=f(x)-g(x) r(x)$ having $2 n+2$ alternate changes of sign in $[1, b]$, one may still ask whether there exists an optimal irreducible rational polynomial of the same degree but its $\delta(x)$ has a smaller number of alternate changes of sign in $[1, b]$. Such a possibility is ruled out by Chebyshev's theorem, which can be shown as follows.

Again, our strategy is to assume the contrary is true, and then show that it leads to contradiction. Suppose there exists an optimal rational polynomial $R(x)=P(x) / Q(x)$ of degree $n$ with deviation $d(R)=d_{\min }$, but its $\Delta(x) \equiv f(x)$ $-g(x) R(x)$ only has $m=2 n+1$ alternate changes of sign in $[1, b]$. Now assume $\Delta(x)$ attains its maxima and minima, say,

$$
\Delta(x)=\Delta_{1},-\Delta_{2},+\Delta_{3}, \ldots,+\Delta_{m}\left(\Delta_{i}>0\right),
$$

at consecutive points ${ }^{3}$

\footnotetext{
${ }^{3}$ Without loss of generality, we set $x_{1}=1$ and $x_{m}=b$.
} 


$$
1=x_{1}<x_{2}<\cdots<x_{m}=b .
$$

Then the interval $[1, b]$ can be divided into $m$ subintervals,

$$
\left[1, \xi_{1}\right],\left[\xi_{1}, \xi_{2}\right], \ldots,\left[\xi_{m-1}, b\right], \quad x_{i}<\xi_{i}<x_{i+1}
$$

such that the following two inequalities can be satisfied alternately:

$$
\begin{gathered}
-d_{\text {min }}+\epsilon<\Delta(x) \leqslant d_{\text {min }}, \\
x \in\left[\xi_{2 i-2}, \xi_{2 i-1}\right], i=1, \ldots, n+1, \\
-d_{\text {min }} \leqslant \Delta(x)<d_{\text {min }}-\epsilon, \quad x \in\left[\xi_{2 i-1}, \xi_{2 i}\right], i=1, \ldots, n,
\end{gathered}
$$

where $\epsilon$ is a positive number which is much less than $d_{\min }$, and the notations $\xi_{0}=1$ and $\xi_{m}=b$ have been used.

Next consider the following polynomial of degree $2 n$ $=m-1$ :

$$
S(x)=\left(x-\xi_{1}\right)\left(x-\xi_{2}\right) \cdots\left(x-\xi_{m-1}\right)
$$

which has sign changes at $\xi_{i}, i=1, \ldots, m-1$. Using the fact that $P(x)$ and $Q(x)$ have no common factor, one can find polynomials $U(x)$ and $V(x)$ of degree $n$, with positive real coefficients, such that

$$
S(x)=Q(x) U(x)-P(x) V(x) .
$$

Now consider the following irreducible rational polynomial of degree $n$

$$
W(x)=\frac{P(x)+\alpha U(x)}{Q(x)+\alpha V(x)}
$$

where $\alpha$ is a positive real parameter. Then, the difference

$$
\begin{aligned}
f(x)-g(x) W(x) & =f(x)-g(x) R(x)+g(x) R(x)-g(x) W(x) \\
& =\Delta(x)-\alpha g(x) \frac{S(x)}{Q(x)[Q(x)+\alpha V(x)]}
\end{aligned}
$$

is a function of $\alpha$ which can be chosen to be some tiny positive number such that the magnitude of the second term on the right-hand side (RHS) of Eq. (44) is much less than $d_{\min }-\epsilon$ for all $x \in[1, b]$, i.e.,

$$
0<\alpha g(x) \frac{|S(x)|}{Q(x)[Q(x)+\alpha V(x)]} \leqslant \mu \ll d_{\text {min }}-\epsilon .
$$

Since $\Delta(x)$ and $S(x)$ both have $m=2 n+1$ alternate changes of sign in $[1, b]$, and the magnitude of the second term on the RHS of Eq. (44) is very small compared to $d_{\text {min }}-\epsilon$, it follows that $f(x)-g(x) W(x)$ also has $m=2 n$ +1 alternate changes of sign in $[1, b]$.

Then, using Eqs. (39), (41), (44), and (45), we obtain

$$
-d_{\text {min }}+\epsilon-\mu<f(x)-g(x) W(x) \leqslant d_{\text {min }}-\mu
$$

for $x \in\left[\xi_{2 i-2}, \xi_{2 i-1}\right], i=1, \ldots, n+1$. Similarly, we have
TABLE I. The error $\sigma$ [Eq. (56)] of the matrix-vector multiplication $H_{w}\left(H_{w}^{2}\right)_{Z}^{-1 / 2} Y$, for several gauge configurations on three different lattices. Evidently, each $\sigma$ is considerably smaller than the corresponding theoretical error bound $2 d_{Z}(n, b)=2(1-\lambda) /(1$ $+\lambda)$.

Lattice

$\begin{array}{lllllllll}\text { size } & \beta & \lambda_{\min } & \lambda_{\max } & b & n & \sigma & 2 d_{Z}(n, b)\end{array}$

$\begin{array}{llllllll}16^{3} \times 32 & 6.0 & 0.1731 & 6.258 & 1307.00 & 12 & 6.0 \times 10^{-12} & 1.4 \times 10^{-10}\end{array}$ $\begin{array}{llllllll}16^{3} \times 32 & 6.0 & 0.1943 & 6.260 & 1038.01 & 12 & 7.0 \times 10^{-12} & 7.5 \times 10^{-11}\end{array}$ $\begin{array}{llllllll}16^{3} \times 32 & 6.0 & 0.1767 & 6.261 & 1255.49 & 12 & 8.0 \times 10^{-12} & 1.2 \times 10^{-10}\end{array}$ $\begin{array}{llllllll}16^{3} \times 32 & 6.0 & 0.1955 & 6.260 & 1025.31 & 12 & 5.0 \times 10^{-12} & 7.2 \times 10^{-11}\end{array}$ $\begin{array}{llllllll}12^{3} \times 24 & 5.8 & 0.1176 & 6.210 & 2788.49 & 16 & 7.0 \times 10^{-14} & 4.8 \times 10^{-13}\end{array}$ $\begin{array}{llllllll}12^{3} \times 24 & 5.8 & 0.1285 & 6.211 & 2336.24 & 16 & 6.6 \times 10^{-14} & 3.2 \times 10^{-13}\end{array}$ $\begin{array}{llllllll}12^{3} \times 24 & 5.8 & 0.0988 & 6.206 & 3945.57 & 16 & 2.0 \times 10^{-13} & 1.3 \times 10^{-12}\end{array}$ $\begin{array}{llllllll}12^{3} \times 24 & 5.8 & 0.1415 & 6.213 & 1927.92 & 16 & 5.5 \times 10^{-14} & 1.6 \times 10^{-13}\end{array}$ $\begin{array}{llllllll}10^{3} \times 24 & 5.8 & 0.1242 & 6.214 & 2503.22 & 16 & 1.6 \times 10^{-13} & 3.4 \times 10^{-13}\end{array}$ $\begin{array}{llllllll}10^{3} \times 24 & 5.8 & 0.1381 & 6.209 & 2021.42 & 16 & 6.1 \times 10^{-14} & 2.2 \times 10^{-13}\end{array}$ $\begin{array}{llllllll}10^{3} \times 24 & 5.8 & 0.1376 & 6.204 & 2032.86 & 16 & 5.5 \times 10^{-14} & 2.6 \times 10^{-13}\end{array}$

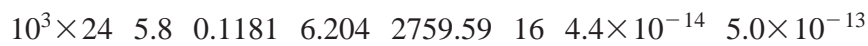

$$
-d_{\text {min }}+\mu \leqslant f(x)-g(x) W(x) \leqslant d_{\text {min }}-\epsilon+\mu
$$

for $x \in\left[\xi_{2 i-1}, \xi_{2 i}\right], i=1, \ldots, n$. Thus we obtain that $d(W)$ $=d_{\text {min }}-\mu<d_{\text {min }}$, a contradiction of our assumption that $R(x)$ is the optimal one. This completes the proof of Chebyshev's theorem.

In general, Chebyshev's theorem asserts that the optimal irreducible rational polynomial of the form (15) must satisfy the criterion that the difference $\delta(x)=f(x)-g(x) r^{(n, m)}(x)$ has $n+m+2$ alternate changes of sign in the interval $[1, b]$.

\section{ZOLOTAREV APPROXIMATION FOR THE OVERLAP}

In this section, we first derive the theoretical error bound for the the matrix-vector multiplication $H_{w}\left(H_{w}^{2}\right)^{-1 / 2} Y(Y$ is any nonzero column vector) with the Zolotarev approximation for $\left(H_{w}^{2}\right)^{-1 / 2}$. Then the numerical values of the error bound are computed, and listed in Table I, as well as plotted in Fig. 3. An empirical formula for the error bound is determined from the data.

\section{A. Error bound for $H_{w}\left(H_{w}^{2}\right)_{Z}^{-1 / 2} Y$}

To use the Zolotarev approximation for $\left(H_{w}^{2}\right)^{-1 / 2}$ in the overlap Dirac operator (1), one needs to rescale $H_{w}$ to $h_{w}$ $=H_{w} / \lambda_{\min }$ such that the eigenvalues of $h_{w}^{2}$ fall in the interval $[1, b]$, where $b=\left(\lambda_{\max } / \lambda_{\min }\right)^{2}$. Explicitly,

\footnotetext{
${ }^{4}$ Here we emphasize that $d_{0}$ [Eq. (49)] can be obtained explicitly, without using the condition $\left.\max \left[1-\sqrt{x} r_{n}(x)\right]\right|_{1 \leqslant x \leqslant b}=-\min [1$ $\left.-\sqrt{x} r_{n}(x)\right]\left.\right|_{1 \leqslant x \leqslant b}$.
} 


\section{$\log _{10}\left[d\left(r_{z}^{(n, n)}\right)\right]$}

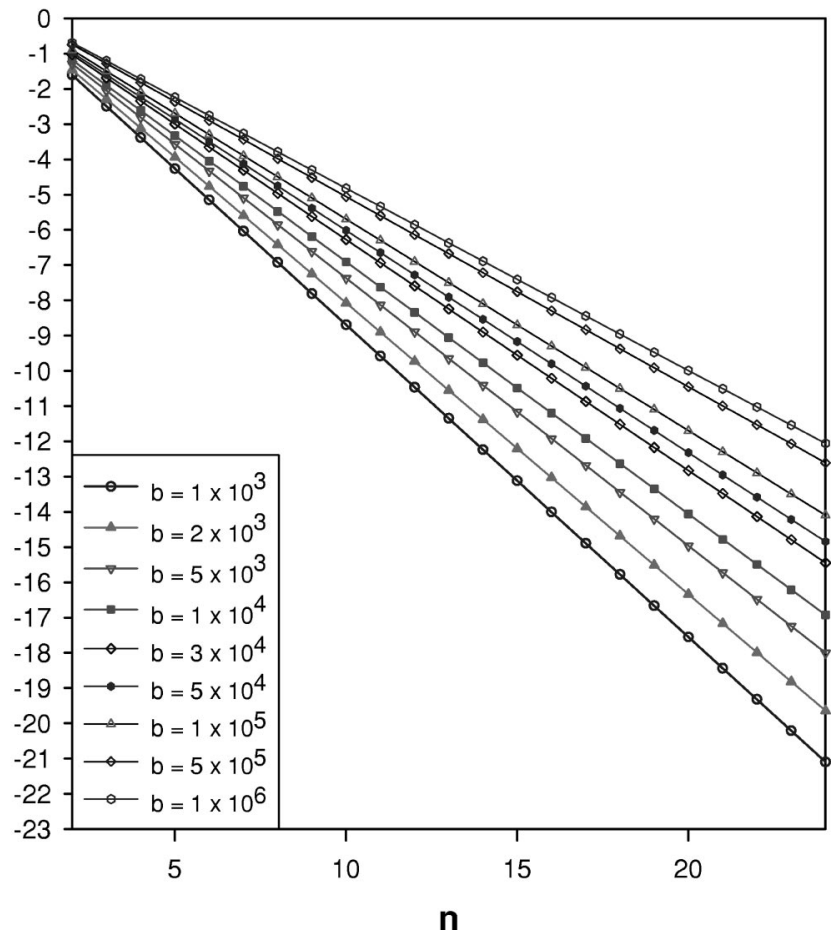

FIG. 3. The error bound of the Zolotarev optimal rational approximation for the inverse square root function $x^{-1 / 2}, x \in[1, b]$, versus the degree $n$ of the rational polynomial, and for several different values of $b$ ranging from $10^{3}$ to $10^{5}$. For any fixed value of $b$, the error bound converges exponentially with respect to $n$, and is well fitted by $A(b) \exp \{-c(b) n\}$ with $c(b)$ $=9.17(10) \ln (b)^{-0.774(5)}$ and $A(b)=0.465(9) \ln (b)^{0.596(9)}$.

$$
\begin{aligned}
\frac{1}{\sqrt{H_{w}^{2}}} & \simeq \frac{d_{0}}{\lambda_{\min } l=1} \prod_{w}^{n} \frac{h_{w}^{2}+c_{2 l}}{h_{w}^{2}+c_{2 l-1}} \\
& =\frac{1}{\lambda_{\min }}\left(h_{w}^{2}+c_{2 n}\right) \sum_{l=1}^{n} \frac{b_{l}}{h_{w}^{2}+c_{2 l-1}} \\
& \equiv\left(H_{w}^{2}\right)_{Z}^{-1 / 2}
\end{aligned}
$$

where

$$
\begin{aligned}
& d_{0}=\frac{2 \lambda}{1+\lambda} \prod_{l=1}^{n} \frac{1+c_{2 l-1}}{1+c_{2 l}}, \\
& b_{l}=d_{0} \frac{\prod_{i=1}^{n-1}\left(c_{2 i}-c_{2 l-1}\right)}{\prod_{i=1, i \neq l}^{n}\left(c_{2 i-1}-c_{2 l-1}\right)} .
\end{aligned}
$$

First, we consider the multiplication of $H_{w}\left(H_{w}^{2}\right)^{-1 / 2}$ by a nonzero column vector $Y$

$$
\begin{aligned}
H_{w}\left(\frac{1}{\sqrt{H_{w}^{2}}}\right) Y & \simeq h_{w}\left(h_{w}^{2}+c_{2 n}\right) \sum_{l=1}^{n} \frac{b_{l}}{h_{w}^{2}+c_{2 l-1}} Y \\
& =h_{w}\left(h_{w}^{2}+c_{2 n}\right) \sum_{l=1}^{n} b_{l} Z_{l}
\end{aligned}
$$

where the last summation can be evaluated by invoking a conjugate gradient process for the linear systems

$$
\left(h_{w}^{2}+c_{2 l-1}\right) Z_{l}=Y, \quad l=1, \ldots, n .
$$

In order to improve the accuracy of the rational approximation as well as to reduce the number of iterations in the conjugate gradient loop, it is advantageous to narrow the interval $[1, b]$ by projecting out the largest and some lowlying eigenmodes of $H_{w}^{2}$. Denoting these eigenmodes by

$$
H_{w} u_{j}=\lambda_{j} u_{j}, \quad j=1, \ldots, k,
$$

we project the linear systems (52) to the complement of the vector space spanned by these eigenmodes,

$$
\left(h_{w}^{2}+c_{2 l-1}\right) \bar{Z}_{l}=\bar{Y} \equiv\left(1-\sum_{j=1}^{k} u_{j} u_{j}^{\dagger}\right) Y, \quad l=1, \ldots, n .
$$

In the set of projected eigenvalues of $H_{w}^{2}\left\{\lambda_{j}^{2}, j\right.$ $=1, \ldots, k\}$, we use $\lambda_{\text {max }}^{2}$ and $\lambda_{\text {min }}^{2}$ to denote the least upper bound and the greatest lower bound for the eigenvalues of $\bar{H}_{w}^{2}$, where

$$
\bar{H}_{w}=H_{w}-\sum_{j=1}^{k} \lambda_{j} u_{j} u_{j}^{\dagger}
$$

Then the eigenvalues of

$$
h_{w}^{2}=\bar{H}_{w}^{2} / \lambda_{\min }^{2}
$$

fall into the interval $(1, b), b=\left(\lambda_{\max } / \lambda_{\min }\right)^{2}$.

Now the matrix-vector multiplication (51) can be expressed in terms of the projected eigenmodes (53) plus the solution obtained from the conjugate gradient loop (54) in the complementary vector space, i.e.,

$$
\begin{aligned}
H_{w} \frac{1}{\sqrt{H_{w}^{2}}} Y \simeq & \frac{1}{\lambda_{\min }} \bar{H}_{w}\left(h_{w}^{2}+c_{2 n}\right) \sum_{l=1}^{n} b_{l} \bar{Z}_{l} \\
& +\sum_{j=1}^{k} \frac{\lambda_{j}}{\sqrt{\lambda_{j}^{2}}} u_{j} u_{j}^{\dagger} Y \equiv S .
\end{aligned}
$$

Then the error of $S$ can be measured in terms of

$$
\sigma=\frac{\left|S^{\dagger} S-Y^{\dagger} Y\right|}{Y^{\dagger} Y}
$$

which is zero if Eq (55) is exact. Now assuming that the errors due to the conjugate gradient and the projected eigenmodes are negligible compared with that due to the Zolo- 
tarev approximation of $\left(h_{w}^{2}\right)^{-1 / 2}$, then we can derive the theoretical upper bound of $\sigma$ as follows.

First, we rewrite $S$ [Eq. (55)] as

$$
\begin{gathered}
S=\sum_{j=1}^{k} \frac{\lambda_{j}}{\sqrt{\lambda_{j}^{2}}} u_{j} u_{j}^{\dagger} Y+\sum_{j=k+1}^{N} \operatorname{sgn}\left(\lambda_{j}\right) \sqrt{x_{j}} r_{Z}\left(x_{j}\right) u_{j} u_{j}^{\dagger} Y, \\
x_{j} \equiv\left(\frac{\lambda_{j}}{\lambda_{\text {min }}}\right)^{2},
\end{gathered}
$$

where $\lambda_{j}, u_{j}, j=k+1, \ldots, N$, denote the eigenvalues and eigenfunctions of $\bar{H}_{w}$. Then it is straightforward to derive

$$
S^{\dagger} S-Y^{\dagger} Y=Y^{\dagger}\left(\sum_{j=k+1}^{N}\left\{\left[\sqrt{x_{j}} r_{Z}\left(x_{j}\right)\right]^{2}-1\right\} u_{j} u_{j}^{\dagger}\right) Y,
$$

where the orthonormality $\left(u_{i}^{\dagger} u_{j}=\delta_{i j}\right)$ has been used. Thus it follows that

$$
\begin{aligned}
\left|S^{\dagger} S-Y^{\dagger} Y\right| \leqslant & \max _{k+1 \leqslant j \leqslant N}\left|\left[\sqrt{x_{j}} r_{Z}\left(x_{j}\right)\right]^{2}-1\right| Y^{\dagger}\left(\sum_{l=k+1}^{N} u_{l} u_{l}^{\dagger}\right) Y \\
& <\max _{k+1 \leqslant j \leqslant N}\left|\left[\sqrt{x_{j}} r_{Z}\left(x_{j}\right)\right]^{2}-1\right| Y^{\dagger} Y
\end{aligned}
$$

where the inequality (due to the completeness relation $\left.\sum_{l=1}^{N} u_{l} u_{l}^{\dagger}=\mathbf{1}\right)$

$$
Y^{\dagger}\left(\sum_{l=k+1}^{N} u_{l} u_{l}^{\dagger}\right) Y<Y^{\dagger} Y, \quad(k>0)
$$

has been used. Then the theoretical upper bound of [Eq. (56)] is

$$
\sigma<\max _{k+1 \leqslant j \leqslant N}\left|\left[\sqrt{x_{j}} r_{Z}\left(x_{j}\right)\right]^{2}-1\right| \simeq 2 \max _{k+1 \leqslant j \leqslant N}\left|\sqrt{x_{j}} r_{Z}\left(x_{j}\right)-1\right| .
$$

Thus $\sigma$ is less than twice the maximum of $\left|1-\sqrt{x_{j}} r_{Z}\left(x_{j}\right)\right|$ for all eigenvalues of $h_{w}^{2}$. It follows that $\sigma$ must be less than twice the maximum of $\left|1-\sqrt{x} r_{Z}(x)\right|$ for all $x \in[1, b]$, i.e.,

$$
\sigma=\frac{\left|S^{\dagger} S-Y^{\dagger} Y\right|}{Y^{\dagger} Y}<\frac{2(1-\lambda)}{1+\lambda} \equiv 2 d_{Z}(n, b),
$$

where $\lambda$ is defined in Eq. (22). The inequality (61) is one of the main results of this paper.

A remarkable feature of Eq. (61) is that it holds for any nonzero column vector $Y$. Then one immediately sees that the deviation (6) which measures the chiral symmetry breaking due to the Zolotarev approximation also has the same theoretical upper bound,

$$
\Delta_{Z}<2 d_{Z}(n, b) \text {. }
$$

Thus $2 d_{Z}(n, b)$ not only serves as the theoretical error bound for the matrix-vector multiplication $H_{w}\left(H_{w}^{2}\right)_{Z}^{-1 / 2} Y$, but also provides the upper bound for the chiral symmetry breaking due to the Zolotarev approximation. Note that $d_{Z}(n, b)$ does not depend on the lattice size explicitly. Therefore, by choosing the proper values of $n$ and $b$ (i.e., by projecting the highand low-lying eigenmodes of $H_{w}^{2}$ ), one can essentially preserve the exact chiral symmetry of the overlap Dirac operator to very high precision, for any gauge configurations on a finite lattice.

Moreover, for any gauge configuration, it is unlikely that any of the eigenvalues of $h_{w}^{2}$ would coincide with one of those $2 n+2$ positions with maximum deviation (34); thus one usually obtains a $\sigma$ much smaller than the theoretical error bound $2 d_{Z}(n, b)$.

In Table I, we list the values of $\sigma$ [Eq. (56)] for several gauge configurations on three different lattices, along with the theoretical error bound $2 d_{Z}(n, b)$. It is clear that $\sigma$ is always much smaller than the theoretical error bound. Note that for each configuration we show only the largest $\sigma$ among a set of several hundred $\sigma$ values; each is computed with a different $Y$ at every iteration of the outer conjugate gradient (CG) loop. The average value of $\sigma$ is usually less than 1/5 of the largest one listed in Table I. Details of our computation are described in [9]. Here we have set the stopping criterion for the inner and outer conjugate gradient loops at $\epsilon=10^{-11}$, and the error of the projected eigenmodes around $10^{-13}$. It is remarkable that $\sigma$ turns out to be much less than $\epsilon$, in contrast to one's naive expectation. In other words, even if the precision of the overlap Dirac quark propagator is only up to $10^{-11}$, its exact chiral symmetry can attain $\Delta_{Z}<10^{-12}$.

\section{B. An empirical formula for the error bound}

In Table II, we list the values of $d_{Z}(n, b)=(1-\lambda) /(1$ $+\lambda)$ of the Zolotarev optimal rational appproximation (29), for $n \simeq 10-20$ and $b \simeq 10-10^{6}$. We can use Table II to determine how many Zolotarev terms $(n)$ are needed in order to attain one's desired accuracy, after the highest- and the lowlying eigenmodes are projected out and $b=\left(\lambda_{\text {max }} / \lambda_{\text {min }}\right)^{2}$ has been obtained. Conversely, for a fixed number of Zolotarev terms, say $n$, one can use Table II to determine what ranges of high- and low-lying eigenmodes of $H_{w}^{2}$ should be projected in order to attain one's desired accuracy.

In Fig. 3, we plot $d_{Z}(n, b)=\max \left|1-\sqrt{x} r_{Z}^{(n, n)}(x)\right|=(1$ $-\lambda) /(1+\lambda)$ versus the degree $n$, for different values of $b$ ranging from $10^{3}-10^{6}$. For any fixed value of $b$, the error bound converges exponentially with respect to $n$, and it is well fitted by

$$
e_{Z}(n, b)=A(b) \exp \{-c(b) n\}
$$

as indicated by the solid lines shown in Fig. 3. The parameters $c(b)$ and $A(b)$ are determined in Figs. 4 and 5, respectively, 
TABLE II. The deviation $d_{Z}(n, b)=\max \left|1-\sqrt{x} r_{Z}^{(n, n)}(x)\right|=(1-\lambda) /(1+\lambda)$ of the Zolotarev optimal rational approximation (29), versus the upper bound $b$ of $x \in[1, b]$, and the degree $n$ of the rational polynomial.

\begin{tabular}{|c|c|c|c|c|c|c|}
\hline \multirow[t]{2}{*}{$b$} & \multicolumn{6}{|c|}{$n$} \\
\hline & 10 & 12 & 14 & 16 & 18 & 20 \\
\hline 10 & $4.8 \times 10^{-18}$ & $1.9 \times 10^{-21}$ & $7.2 \times 10^{-25}$ & $2.8 \times 10^{-28}$ & $1.1 \times 10^{-31}$ & $4.1 \times 10^{-35}$ \\
\hline 50 & $1.3 \times 10^{-13}$ & $3.5 \times 10^{-16}$ & $9.5 \times 10^{-19}$ & $2.6 \times 10^{-21}$ & $6.9 \times 10^{-24}$ & $1.9 \times 10^{-26}$ \\
\hline 100 & $2.5 \times 10^{-12}$ & $1.2 \times 10^{-14}$ & $5.5 \times 10^{-17}$ & $2.6 \times 10^{-19}$ & $1.2 \times 10^{-21}$ & $5.8 \times 10^{-24}$ \\
\hline 500 & $3.8 \times 10^{-10}$ & $4.8 \times 10^{-12}$ & $5.9 \times 10^{-14}$ & $7.3 \times 10^{-16}$ & $9.0 \times 10^{-18}$ & $1.1 \times 10^{-19}$ \\
\hline 1000 & $2.0 \times 10^{-9}$ & $3.4 \times 10^{-11}$ & $5.8 \times 10^{-13}$ & $9.8 \times 10^{-15}$ & $1.7 \times 10^{-16}$ & $2.8 \times 10^{-18}$ \\
\hline 2000 & $8.4 \times 10^{-9}$ & $1.9 \times 10^{-10}$ & $4.2 \times 10^{-12}$ & $9.3 \times 10^{-14}$ & $2.1 \times 10^{-15}$ & $4.6 \times 10^{-17}$ \\
\hline 3000 & $1.8 \times 10^{-8}$ & $4.6 \times 10^{-10}$ & $1.2 \times 10^{-11}$ & $3.0 \times 10^{-13}$ & $7.7 \times 10^{-15}$ & $2.0 \times 10^{-16}$ \\
\hline 4000 & $2.9 \times 10^{-8}$ & $8.3 \times 10^{-10}$ & $2.3 \times 10^{-11}$ & $6.6 \times 10^{-13}$ & $1.9 \times 10^{-14}$ & $5.3 \times 10^{-16}$ \\
\hline 5000 & $4.3 \times 10^{-8}$ & $1.3 \times 10^{-9}$ & $3.9 \times 10^{-11}$ & $1.2 \times 10^{-12}$ & $3.6 \times 10^{-14}$ & $1.1 \times 10^{-15}$ \\
\hline 6000 & $5.7 \times 10^{-8}$ & $1.8 \times 10^{-9}$ & $5.8 \times 10^{-11}$ & $1.9 \times 10^{-12}$ & $6.0 \times 10^{-14}$ & $1.9 \times 10^{-15}$ \\
\hline 7000 & $7.2 \times 10^{-8}$ & $2.4 \times 10^{-9}$ & $8.1 \times 10^{-11}$ & $2.7 \times 10^{-12}$ & $9.1 \times 10^{-14}$ & $3.1 \times 10^{-15}$ \\
\hline 8000 & $8.9 \times 10^{-8}$ & $3.1 \times 10^{-9}$ & $1.1 \times 10^{-10}$ & $3.7 \times 10^{-12}$ & $1.3 \times 10^{-13}$ & $4.5 \times 10^{-15}$ \\
\hline 9000 & $1.1 \times 10^{-7}$ & $3.8 \times 10^{-9}$ & $1.4 \times 10^{-10}$ & $4.9 \times 10^{-12}$ & $1.8 \times 10^{-13}$ & $6.4 \times 10^{-15}$ \\
\hline $1 \times 10^{4}$ & $1.2 \times 10^{-7}$ & $4.6 \times 10^{-9}$ & $1.7 \times 10^{-10}$ & $6.3 \times 10^{-12}$ & $2.3 \times 10^{-13}$ & $8.6 \times 10^{-15}$ \\
\hline $5 \times 10^{4}$ & $9.5 \times 10^{-7}$ & $5.2 \times 10^{-8}$ & $2.9 \times 10^{-9}$ & $1.6 \times 10^{-10}$ & $8.6 \times 10^{-12}$ & $4.7 \times 10^{-13}$ \\
\hline $1 \times 10^{5}$ & $2.0 \times 10^{-6}$ & $1.3 \times 10^{-7}$ & $8.0 \times 10^{-9}$ & $5.0 \times 10^{-10}$ & $3.2 \times 10^{-11}$ & $2.0 \times 10^{-12}$ \\
\hline $5 \times 10^{5}$ & $8.7 \times 10^{-6}$ & $7.3 \times 10^{-7}$ & $6.1 \times 10^{-8}$ & $5.0 \times 10^{-9}$ & $4.2 \times 10^{-10}$ & $3.5 \times 10^{-11}$ \\
\hline $1 \times 10^{6}$ & $1.5 \times 10^{-5}$ & $1.4 \times 10^{-6}$ & $1.3 \times 10^{-7}$ & $1.2 \times 10^{-8}$ & $1.1 \times 10^{-9}$ & $1.0 \times 10^{-10}$ \\
\hline
\end{tabular}

$c(b)=9.17(10) \ln (b)^{-0.774(5)}$,

$A(b)=0.465(9) \ln (b)^{0.596(9)}$.

With the empirical formula (63), one can estimate the theoretical upper bound of $\sigma$ or $\Delta_{Z}$ more conveniently, without evaluating ellitpic functions at all.

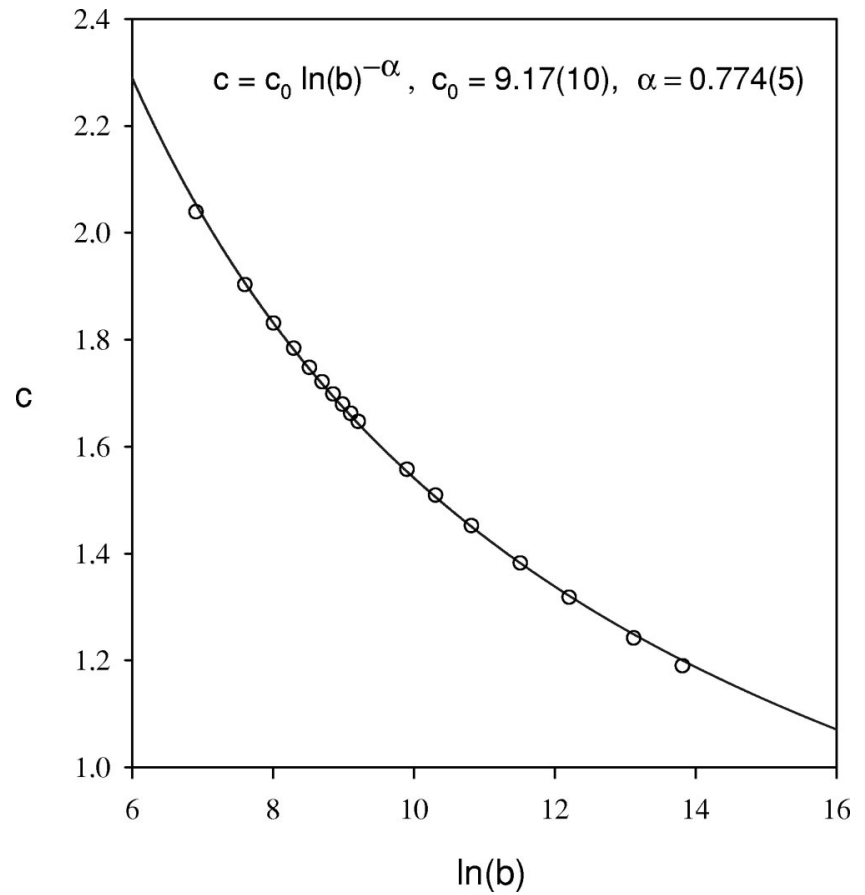

FIG. 4. Determination of the parameters of $c(b)$.

\section{Zolotarev rational polynomial of the form $r^{(n-1, n)}$}

At this point, if one recalls Chebyshev's therorem for $r^{(n, m)}$, one may ask whether there exists an optimal rational approximation to $x^{-1 / 2}$, which has the form $r^{(n-1, n)}$, with $1-\sqrt{x} r_{Z}^{(n-1, n)}(x)$ having $2 n+1$ alternate changes of sign in $[1, b]$. Note that, in the second principal $n$th degree transformation $(21), \operatorname{sn}(x / M ; \lambda)$ has periods $2 K / M$ and $2 i K^{\prime} /[(2 n+1) M]$. Thus there must exist a similar transfor-

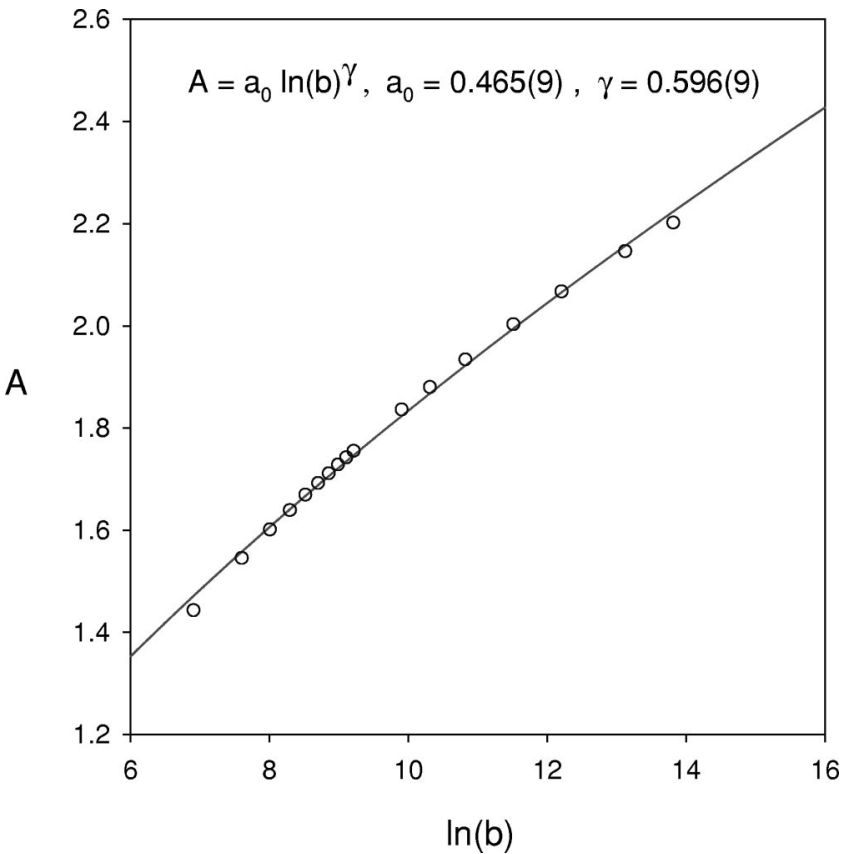

FIG. 5. Determination of the parameters of $A(b)$. 
TABLE III. The deviation $d\left(r_{Z}^{(n-1, n)}\right)=\max \left|1-\sqrt{x} r_{Z}^{(n-1, n)}(x)\right|=(1-\Lambda) /(1+\Lambda)$ of the Zolotarev optimal rational approximation (70) versus the upper bound $b$ of $x \in[1, b]$, and the degree $n$ of the rational polynomial.

\begin{tabular}{lcccccc}
\hline \hline$b$ & & \multicolumn{2}{c}{$n$} & & \\
& 10 & 12 & 14 & 16 & 18 & 20 \\
\hline 1000 & $5.6 \times 10^{-9}$ & $9.4 \times 10^{-11}$ & $1.6 \times 10^{-12}$ & $2.7 \times 10^{-14}$ & $4.6 \times 10^{-16}$ & $7.8 \times 10^{-18}$ \\
2000 & $2.2 \times 10^{-8}$ & $4.8 \times 10^{-10}$ & $1.1 \times 10^{-11}$ & $2.4 \times 10^{-13}$ & $5.3 \times 10^{-15}$ & $1.2 \times 10^{-16}$ \\
3000 & $4.5 \times 10^{-8}$ & $1.1 \times 10^{-9}$ & $2.9 \times 10^{-11}$ & $7.5 \times 10^{-13}$ & $1.9 \times 10^{-14}$ & $5.0 \times 10^{-16}$ \\
4000 & $7.2 \times 10^{-8}$ & $2.0 \times 10^{-9}$ & $5.7 \times 10^{-11}$ & $1.6 \times 10^{-12}$ & $4.6 \times 10^{-14}$ & $1.3 \times 10^{-15}$ \\
5000 & $1.0 \times 10^{-7}$ & $3.1 \times 10^{-9}$ & $9.4 \times 10^{-11}$ & $2.8 \times 10^{-12}$ & $8.6 \times 10^{-14}$ & $2.6 \times 10^{-15}$ \\
6000 & $1.3 \times 10^{-7}$ & $4.3 \times 10^{-9}$ & $1.4 \times 10^{-10}$ & $4.4 \times 10^{-12}$ & $1.4 \times 10^{-13}$ & $4.5 \times 10^{-15}$ \\
7000 & $1.7 \times 10^{-7}$ & $5.7 \times 10^{-9}$ & $1.9 \times 10^{-10}$ & $6.4 \times 10^{-12}$ & $2.1 \times 10^{-13}$ & $7.2 \times 10^{-15}$ \\
8000 & $2.1 \times 10^{-7}$ & $7.1 \times 10^{-9}$ & $2.5 \times 10^{-10}$ & $8.7 \times 10^{-12}$ & $3.0 \times 10^{-13}$ & $1.1 \times 10^{-14}$ \\
9000 & $2.4 \times 10^{-7}$ & $8.7 \times 10^{-9}$ & $3.1 \times 10^{-10}$ & $1.1 \times 10^{-11}$ & $4.1 \times 10^{-13}$ & $1.5 \times 10^{-14}$ \\
10000 & $2.8 \times 10^{-7}$ & $1.0 \times 10^{-8}$ & $3.9 \times 10^{-10}$ & $1.4 \times 10^{-11}$ & $5.3 \times 10^{-13}$ & $2.0 \times 10^{-14}$ \\
\hline
\end{tabular}

mation in which $\operatorname{sn}(x / M ; \lambda)$ has periods $2 K / M$ and $2 i K^{\prime} / 2 n M$. Explicitly, it reads

$$
\sqrt{x\left(\frac{u}{m} ; \Lambda\right)}=\sqrt{x(u ; \kappa)} \frac{1}{m} \frac{\prod_{l=1}^{n-1}\left[1+x(u ; \kappa) / C_{2 l}\right]}{\prod_{l=1}^{n}\left[1+x(u ; \kappa) / C_{2 l-1}\right]}
$$

where

$$
\begin{aligned}
& \Lambda=\prod_{l=1}^{2 n} \frac{\Theta^{2}\left(2 l K^{\prime} / 2 n ; \kappa^{\prime}\right)}{\Theta^{2}\left((2 l-1) K^{\prime} / 2 n ; \kappa^{\prime}\right)}, \\
& m=\frac{\prod_{l=1}^{n} \operatorname{sn}^{2}\left((2 l-1) K^{\prime} / 2 n ; \kappa^{\prime}\right)}{n-1}, \\
& \prod_{l=1} \operatorname{sn}^{2}\left(2 l K^{\prime} / 2 n ; \kappa^{\prime}\right) \\
& C_{l}=\frac{\operatorname{sn}^{2}\left(l K^{\prime} / 2 n ; \kappa^{\prime}\right)}{1-\operatorname{sn}^{2}\left(l K^{\prime} / 2 n ; \kappa^{\prime}\right)} .
\end{aligned}
$$

Then the Zolotarev optimal rational polynomial of the form $r^{(n-1, n)}$ is

$$
r_{Z}^{(n-1, n)}(x)=\frac{2 \Lambda}{1+\Lambda} \frac{1}{m} \frac{\prod_{l=1}^{n-1}\left(1+x / C_{2 l}\right)}{\prod_{l=1}^{n}\left(1+x / C_{2 l-1}\right)} .
$$

The difference $1-\sqrt{x} r_{Z}^{(n-1, n)}(x)$ has $2 n+1$ alternate changes of sign in $[1, b]\left(b=\kappa^{-2}\right)$, and it attains its maxima and minima alternately as

$$
\frac{1-\Lambda}{1+\Lambda},-\frac{1-\Lambda}{1+\Lambda}, \cdots,-\frac{1-\Lambda}{1+\Lambda}, \frac{1-\Lambda}{1+\Lambda}
$$

at $2 n+1$ successive points $x \in[1, b]$ :

$$
\begin{aligned}
& 1, \frac{1}{1-\kappa^{\prime 2} \operatorname{sn}^{2}\left(K^{\prime} / 2 n ; \kappa^{\prime}\right)}, \\
& \cdots, \frac{1}{1-\kappa^{\prime 2} \operatorname{sn}^{2}\left((2 n-1) K^{\prime} / 2 n ; \kappa^{\prime}\right)}, \frac{1}{\kappa^{2}} .
\end{aligned}
$$

Obviously, for any given $n$ and $b$, the deviation of $r_{Z}^{(n-1, n)}$ is larger than that of $r_{Z}^{(n, n)}$, i.e.,

$$
d\left(r_{Z}^{(n-1, n)}\right)=\frac{1-\Lambda}{1+\Lambda}>\frac{1-\lambda}{1+\lambda}=d\left(r_{Z}^{(n, n)}\right)
$$

and for most cases

$$
d\left(r_{Z}^{(n-1, n)}\right) \simeq 2.5 \times d\left(r_{Z}^{(n, n)}\right) .
$$

In Table III, we list the deviation $d\left(r_{Z}^{(n-1, n)}\right)=(1$ $-\Lambda) /(1+\Lambda)$ of the Zolotarev optimal rational appproximation (70), versus the degree $n$, and the upper bound $b$ of $x$ $\in[1, b]$. Comparing the corresponding entries (with the same $b$ and $n$ ) in Table III and Table II, one immediately sees that $d\left(r_{Z}^{(n-1, n)}\right) \simeq 2.5 \times d\left(r_{Z}^{(n, n)}\right)$.

If one uses Eq. (70) to approximate the inverse square root of $H_{w}^{2}$ in the overlap Dirac operator, then one has

$$
\frac{1}{\sqrt{H_{w}^{2}}} \simeq \frac{D_{0}}{\lambda_{\min }} \frac{\prod_{l=1}^{n-1}\left(h_{w}^{2}+C_{2 l}\right)}{\prod_{l=1}^{n}\left(h_{w}^{2}+C_{2 l-1}\right)}=\frac{D_{0}}{\lambda_{\min }} \sum_{l=1}^{n} \frac{B_{l}}{h_{w}^{2}+C_{2 l-1}}
$$

where 


$$
\begin{aligned}
& D_{0}=\frac{2 \Lambda}{1+\Lambda} \frac{\prod_{l=1}^{n}\left(1+C_{2 l-1}\right)}{\prod_{l=1}^{n-1}\left(1+C_{2 l}\right)} \\
& B_{l}=D_{0} \frac{\prod_{i=1}^{n-1}\left(C_{2 i}-C_{2 l-1}\right)}{\prod_{i=1, i \neq l}^{n}\left(C_{2 i-1}-C_{2 l-1}\right)} .
\end{aligned}
$$

Comparing Eq. (48) with Eq. (73), one immediately sees that it is more advantageous to use the former approximation than the latter, especially for computing quark propagators, since only one more matrix multiplication with $\left(h_{w}^{2}+c_{2 n}\right)$ after the completion of the inner CG loop would yield about 2.5 times higher accuracy than using Eq. (73). Although we used Eq. (73) for our computations in Ref. [9], we have switched to Eq. (48) for better accuracy in our ongoing lattice QCD computations.

\section{CONCLUDING REMARKS}

In this paper, we discussed the basic principles underlying the rational approximation, and shown explicitly that the Zolotarev approximation is indeed the optimal rational approximation for the inverse square root function. For the overlap Dirac operator, we derived the theoretical error bound for the matrix-vector multiplication $H_{w}\left(H_{w}^{2}\right)_{Z}^{-1 / 2} Y$, which is equal to twice the maximum deviation $d_{Z}(n, b)$ of the Zolotarev rational polynomial. This is also the upper bound for chiral symmetry breaking due to the Zolotarev approximation. Some numerical values of $d_{Z}(n, b)$ are listed in Table II as well as plotted in Fig. 3. An empirical formula for $d_{Z}(n, b)$ was determined, which provides a reliable estimate of the theoretical error bound, especially for the range of parameters $b \simeq 10^{3}-10^{4}$ and $n \simeq 10-20$. We also compare two possible forms of the Zolotarev optimal rational approximation, $r_{Z}^{(n, n)}(x)$ and $r_{Z}^{(n-1, n)}(x)$, and point out that the former seems to be the better choice for computing quark propagators, since with the same computational cost, one has $d\left(r_{Z}^{(n, n)}\right) \simeq 0.4 \times d\left(r_{Z}^{(n-1, n)}\right)$.

With the Zolotarev optimal rational approximation for $\left(H_{w}^{2}\right)^{-1 / 2}$ in the overlap Dirac operator, one has no difficulties in preserving exact chiral symmetry to very high precision (e.g., $\Delta_{Z}<10^{-12}$ ), for any gauge configurations on a finite lattice. This feature is vital for lattice QCD to extract physical observables from first principles. In practice, one might have difficulties in pushing the error $\sigma$ [Eq. (56)] down below $10^{-13}$, which is essentially due to the inaccuracies of the projected (high- and low-lying) eigenmodes of $H_{w}^{2}$, rather than to the Zolotarev approximation of $\left(H_{w}^{2}\right)^{-1 / 2}$. In the future, we will try to improve the accuracy of the projected eigenmodes. In the meantime, the precision of the exact chiral symmetry up to $10^{-13}$ should be sufficient for many calculations in lattice QCD with overlap Dirac quarks.

\section{ACKNOWLEDGMENT}

This work was supported in part by the National Science Council, ROC, under Grant No. NSC90-2112-M002-021, and also in part by NCTS.
[1] H. Neuberger, Phys. Lett. B 417, 141 (1998).

[2] R. Narayanan and H. Neuberger, Nucl. Phys. B443, 305 (1995).

[3] P. H. Ginsparg and K. G. Wilson, Phys. Rev. D 25, 2649 (1982).

[4] E. I. Zolotarev, Zap. Imp. Akad. Nauk. St. Petersburg, 30, 5 (1877); reprinted in Collected Works (Akademii Nauk SSSR, Moscow, 1932), Vol. 2, pp. 1-59.

[5] N. I. Akhiezer, Theory of Approximation (Dover, New York, 1992).
[6] N. I. Akhiezer, Elements of the Theory of Elliptic Functions, 2nd revised ed. (Nauka, Moscow, 1970) [Mathematical Monographs (American Mathematical Society, Providence, RI, 1990), Vol 79].

[7] D. Ingerman, V. Druskin, and L. Knizhnerman, Commun. Pure Appl. Math. 53, 1039 (2000).

[8] J. van den Eshof, A. Frommer, T. Lippert, K. Schilling, and H. A. van der Vorst, Comput. Phys. Commun. 146, 203 (2002).

[9] T. W. Chiu and T. H. Hsieh, Phys. Rev. D 66, 014506 (2002).

[10] T. W. Chiu and T. H. Hsieh, Phys. Lett. B 538, 298 (2002). 